\title{
THE RINGED X-GALAXY NGC 7020
}

\author{
Ronald Buta \\ Department of Physics and Astronomy, University of Alabama, U.S.A.
}

\section{Introduction}

The southern $\mathrm{S}^{+}$galaxy NGC 7020 presents an unusual morphology: it includes a very regular outer ring which is completely detached and which envelops an inner ring/lens zone with a hexagon surrounding an $\mathrm{X}$ shape (Figures $1 \mathrm{a}$ and $1 \mathrm{~b}$ ). The outer ring has a high contrast compared to those usually observed in barred galaxies, yet NGC 7020 is not obviously barred. The morphology of this galaxy poses an interesting puzzle in that the hexagonal/ $\mathrm{X}$ zone is not a typical type of feature to find in the interior of such a regular ring. Instead, the zone bears a striking resemblance to the edge-on galaxy IC 4767 , recently studied by Whitmore and Bell $(1988=$ WB88 $)$ and dubbed by them as the "X-galaxy" because its inner regions appear to be crossed by two distinct enhancements lined at $\pm 22^{\circ}$ with respect to the major axis. The observation of a similar phenomenon in NGC 7020 is interesting because of the suggestion by WB88 that " $X$ " structures could be related to accretion of matter associated with a merger or tidal encounter between an S0 and a small satellite galaxy. If this interpretation is correct for NGC 7020, then it has important implications for the nature of the outer ring. An alternative interpretation is that the inner hexagonal/ $\mathrm{X}$ zone is a region where resonant periodic orbits in a weak bi-symmetric potential perturbation are influencing the morphology more strongly than might be expected. In this paper, I give a brief summary of a more extensive paper (Buta $1990 \mathrm{c}=\mathrm{B} 90 \mathrm{c})$ and a few other details concerning this interesting galaxy.

\section{Structure}

Details of the hexagonal/X zone are highlighted in Figure $1 \mathrm{~b}$. This image has had a bulge model (B90c) removed in order to enhance the features. A very regular pattern is evident. The inner zone appears crossed by two distinct enhancements that look like an X (see arrows). The arms of the X make angles of $\pm 25^{\circ}$ with respect to the major axis. At the major axis points of the inner zone there are two bright knots (indicated by the letters " $k$ "). These are interesting because they resemble the "ansae" often seen in edge-on S0 galaxies, features which are usually interpreted as due to an inner ring (de Vaucouleurs 1959). However, in a non-edge-on galaxy, such knots must be distinct objects, not artifacts of tilt. A definite boxy character is evident in the region within $10^{\prime \prime}$ of the core. The combination of this region, the $\mathrm{X}$, and the short sides of the hexagon gives 
a diamond-shaped character to the dips in surface brightness at $\pm 23^{\prime \prime}$ from the nucleus along the major axis.

The $\mathrm{X}$ character does not show well on isophote maps, and one could argue that it is not real. In such maps one sees instead a distinctive sequence of isophote shapes, ranging from nearly circular in the core, to elliptical, to rectangular, to hexagonal, to pointed oval (Figure 1d). The map also highlights how two of the shorter sides of the hexagon are brighter than the other two, which gives the impression of a two-armed spiral pattern connecting to the boxy zone.

\section{Properties}

Is there anything unusual about other characteristics of NGC 7020? The absolute blue magnitude is about -20.0 and the outer ring is about $20 \mathrm{kpc}$ in diameter $\left(\mathrm{H}_{0}=100\right.$ scale), neither of which is very extreme. The galaxy is a member of a sparse group having $\left.<V_{\odot}\right\rangle=3,000 \mathrm{~km} \mathrm{~s}^{-1}$, but is not obviously interacting with any other member of that group. After allowance for the more spherical shape of the bulge, Fourier intensity analysis reveals significant $m=2$ amplitude only in the region of the knots, and negligible odd amplitudes. The galaxy is remarkably symmetric. The outer ring appears as a distinct blue enhancement in a color index map (Figure 1c, which is $U-B$ ), suggesting that it is a region with a somewhat more active star formation history than its surroundings (B90c). This is a typical property of most galaxy rings (Buta 1988 and references therein; Buta 1990a,b).

Color index maps like Figure 1c also reveal little color differentiation in the inner hexagonal zone, except for a color gradient in the inner few arcseconds. The knots are found to have colors (integrated over a $4^{\prime \prime}$ aperture) of $\mathrm{B}-\mathrm{V}=0.99$ and $\mathrm{U}-\mathrm{B}=0.55$, compared to 1.04 and 0.63 , respectively, for a $6^{\prime \prime}$ diameter region centered on the nucleus. The knots are therefore made mostly of old stars.

The outer ring contributes only a small fraction of the total luminosity. The enhanced region between $63^{\prime \prime}$ and $91^{\prime \prime}$ radius contributes about $17 \%$ of the total V-band luminosity. A useful parameter for comparing with other galaxies is the luminosity $\left(L_{R}^{B}\right)$ of the ring plus outer disk compared to the luminosity $\left(L_{c}^{B}\right)$ of the inner regions (B-band). These luminosities are obtained by dividing the galaxy at the minimum of the gap region (Wakamatsu 1990). The ratio obtained, $L_{R}^{B} / L_{c}^{B}=0.50$, is comparable to what has been found for normal barred outer-ringed galaxies by Wakamatsu (1990). 


\section{The Nature of the Hexagonal/X Zone}

The most promising interpretation of this zone is that it is related to an unusual type of bar and inner ring. The ratio of the diameter of the outer ring to the separation of the knots is 2.3, very similar to the mean ratio of outer ring to bar diameters in SB galaxies (Kormendy 1979) and the mean ratio of outer to inner ring diameters in double-ringed SB galaxies (Athanassoula et al. 1982). This ratio is also close to expected resonance radius ratios for a velocity $=$ constant rotation curve: $r(\mathrm{OLR}) / \mathrm{r}(\mathrm{CR})=1.7$, where OLR $=$ outer Lindblad resonance and $\mathrm{CR}=$ corotation; $\mathrm{r}(\mathrm{OLR}) / \mathrm{r}(4: 1)=2.6$, where $\mathrm{r}(4: 1)$ is the radius of the inner $4: 1$ resonance; and $r(\mathrm{OLR}) / \mathrm{r}(6: 1)=2.2$, where $r(6: 1)$ is the radius of the inner 6:1 resonance. The observed ratio suggests that the inner zone is possibly associated with the 4:1 and 6:1 ultraharmonic resonances, which could explain the rectangular and hexagonal shapes so evident in that region. However, the sharpness of these features is difficult to understand in a galaxy where no conventional bar is apparent. Either the knots contain enough mass to define an $m=2$ perturbation, or the inner zone is a bar in its own right which is cold enough so that random motions have not washed out some of the finer details of the stellar orbits. An interpretation in terms of planar bar orbits in an integrable galactic model has been discussed by Contopoulos (1988). Vertical resonant orbits may also be important, because these are known to be capable of causing a bar to appear boxy if viewed nearly edge-on (Combes and Sanders 1981; Pfenniger 1984, 1985).

It is more difficult to make a case for the external dynamics interpretation of the inner zone. In this interpretation, a small gas-rich companion is disrupted into an oblique orbit around an S0 galaxy. Before differential precession causes the material to settle into the plane of the S0 disk, the oblique disk spreads into a cone-shaped distribution, which, when viewed edge-on, appears as an X. This could cause the appearance of a "peanut-bulge" as in IC 4767 (WB88). For NGC 7020, the model would require that the outer ring and inner hexagonal zone are not in the same plane, the inner zone being viewed more edgeon. Arguments in favor of the interpretation are the striking resemblance of IC 4767 to the inner zone, both in general appearance and isophote shapes, the group membership of NGC 7020, the similar relative angles of the X-arms of IC 4767 and NGC 7020 with respect to the major axis, and the resemblance of the outer ring to that in Hoag's Object (believed to be of the accretion type by Schweizer et al. 1987). However, arguments against the model are the non-edge-on orientation $\left(i=69^{\circ}\right)$ and obvious outer disk, the similar projected shapes of the inner hexagonal zone and the outer ring, and the lack of evidence for any merger remnant in the core (see WB88). 


\section{CONCLUSIONS}

An unambiguous interpretation of the structure of NGC 7020 will require kinematic and $\mathrm{HI}$ observations. If the hexagonal/ $\mathrm{X}$ zone has been affected by a merger, then the outer ring could itself be an accretion feature, for example, like that in Hoag's Object. This very interesting possibility would imply that the ringed galaxy class does not consist entirely of internally derived structures. Until kinematic and $\mathrm{HI}$ observations are obtained, the present observations tentatively favor the internal dynamics interpretation of the galaxy's structure, that is, that the inner zone is related to a weak bar and inner ring, most likely inside CR, and that the outer ring is related to OLR. The resemblance to IC 4767 may only mean that different mechanisms can lead to a similar observed morphology. However, the problem of how a weak bi-symmetric distortion could have generated orbits with the sharp corners observed needs to be investigated. It is also essential to measure velocity dispersions in the inner zone, since the significant structure would not be expected if this ring/lens zone is as hot as the lenses observed by Kormendy (1984a,b).

\section{References}

Athanassoula, E., Bosma, A., Creze, M., and Schwarz, M. P. 1982, Astr. Ap. 107, 101. Buta, R. 1988, Ap. J. Suppl. 66, 233.

...... 1990a, Ap. J. 351, 62 .

….. 1990b, Ap. J. 354, in press.

….. 1990c, Ap. J. 356, in press (B90c).

Combes, F. and Sanders, R. H. 1981, Astr. Ap. 96, 164.

Contopoulos, G. 1988 in Integrability in Dynamical Systems, Annals of the New York Academy of Sciences, Vol. 596, eds. J. R. Buchler, J. R. Ipser, and C. A. Williams (New York: New York Academy of Sciences), p. 1.

de Vaucouleurs, G. 1959, Handbuch der Physik 53, 275.

Kormendy, J. 1979, Ap. J. 227, 714.

...... 1984a, Ap. J. 286, 116.

…. 1984b, Ap. J. 286, 132.

Pfenniger, D. 1984, Astr. Ap. 134, 373.

…. 1985, Astr. Ap. 150, 112.

Schweizer, F., Ford, W. K., Jedrzejewski, R., and Giovanelli, R. 1987, Ap. J. 320, 454. Wakamatsu, K. 1990, Ap. J. 348, 448.

Whitmore, B. C. and Bell, M. B. 1988, Ap. J. 324, 741 (WB88). 


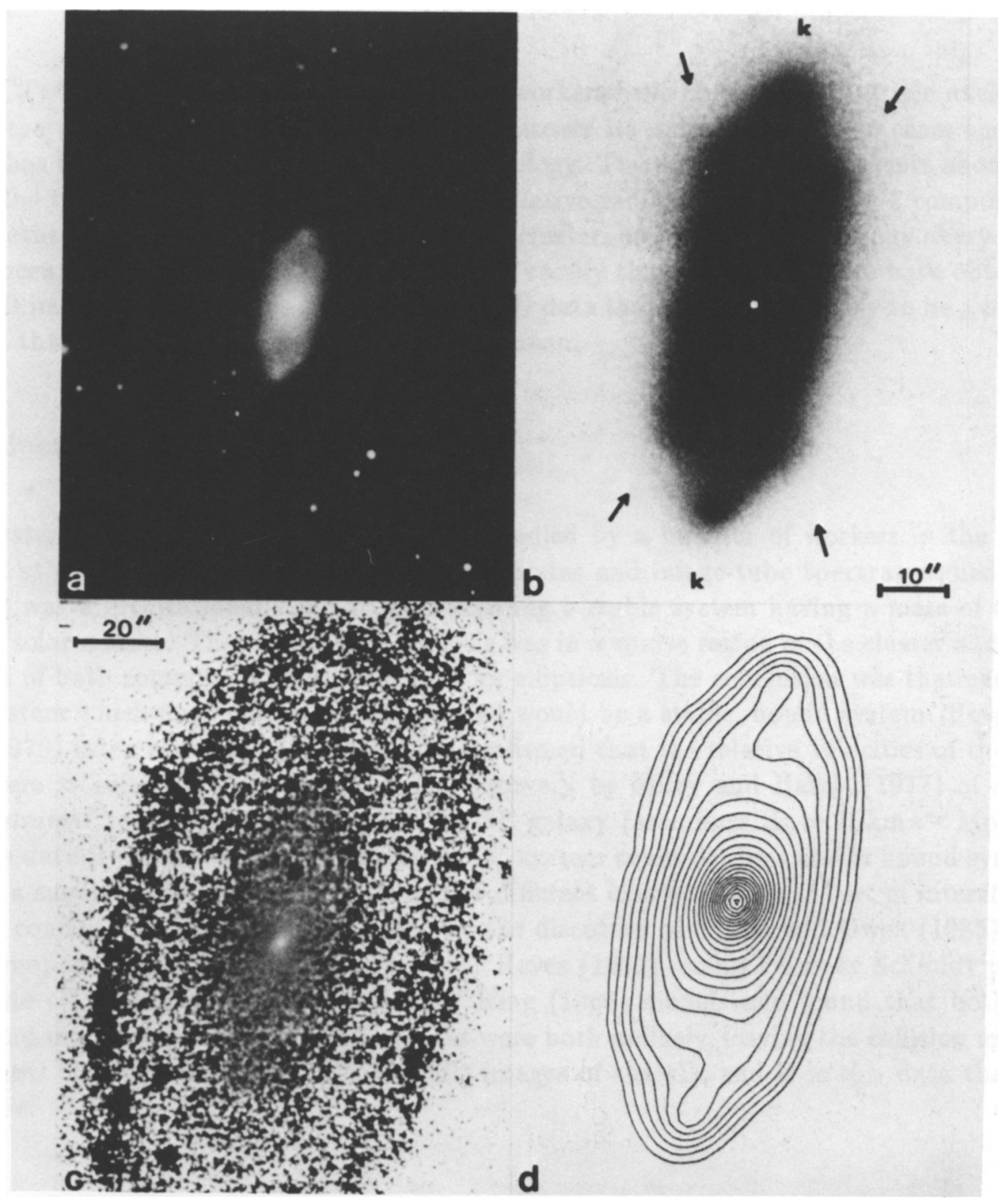

Fig. 1 - Images of NGC 7020: (a) V-band CCD mosaic image (units of mag arcsec ${ }^{-2}$ ); (b) enlargement of inner hexagonal zone ( $V$-band), with arrows indicating the " $X$ " and the letters " $k$ " indicating the knots discussed in text; (c) U-B color index map, coded such that "blue" features are dark while "red" features are light; (d) V-band isophote map showing distinctive sequence of shapes. Largest isophote corresponds to $\mu_{V}=22.37 \mathrm{mag}$ $\operatorname{arcsec}^{-2}$, and each successive isophote is $-0.20 \mathrm{mag} \operatorname{arcsec}^{-2}$ brighter than the previous larger one. Panels (b) and (d) have nearly the same image scale. 